\title{
A Cluster-First Route-Second Heuristic Approach to Solve Periodic Multi-Trip Vehicle Routing Problem
}

\author{
Annisa Kesy Garside *, Nabila Rohmatul Laili \\ Industrial Engineering Department, University of Muhammadiyah Malang, Indonesia \\ Jl. Raya Tlogomas No. 246 Malang, Indonesia \\ * Corresponding author: annisa@umm.ac.id
}

\section{ARTICLE INFO}

Article history

Received January 26, 2019

Revised August 14, 2019

Accepted August 20, 2019

Available Online August 31, 2019

\section{Keywords}

Cluster first route second

Vehicle routing

Multi-trip

Tabu search

Periodic

\begin{abstract}
This paper discusses periodic vehicle routing problems that allow vehicles to travel on multiple trips in a single day. It is known as the Multi-Trip Periodic Vehicles (MTPVRP) Problem Route. Cluster-first route-second (CFRS) heuristics to solve MTPVRP was proposed in this study. In phase 1 , customers were divided into clusters using the formulation of integer programming. Phase 2 determined the route of the cluster and verified that the total journey time to visit the trips does not exceed the working hours of the vehicle. Implementing the heuristic CFRS to solve the real problem faced by the Liquefied petroleum gas (LPG) distributor shows that the procedure could provide a better routing solution.
\end{abstract}

This is an open-access article under the CC-BY-SA license.

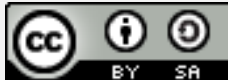

\section{Introduction}

Dantzig and Ramser [1] introduced the Vehicle Routing Problem (VRP) that describes a real-world routing problem of gasoline delivery trucks. Recently, VRP is still an optimization problem that continues to be researched and gets much attention. The state-of-the-art VRP work has been discussed in many survey papers. Some of them are Eksioglu, et al. [2] Lin, et al. [3], and Srivatsa Srinivas and Gajanand [4]. VRP is solved by two methods: exact and heuristic methods [5]. Cluster-first path-second (CFRS) is the most common heuristic approach to solve CVRP. Two early CFRS study was conducted by Gillett and Miller [6] and Fisher and Jaikumar [7]. The CFRS approach is still used up to now because of the simplicity, for example, in research by Dondo and Cerdá [8], Shin and Han [9], Cakir, et al. [10], and Cömsert, Et Al. [11]. Hiquebran, et al. [12] stated that the CFRS approach should provide a better quality solution despite requiring more complex computation than route-first cluster-second (RFCS).

The companies are trying to minimize the number of vehicles due to the costs incurred. Therefore, a vehicle should travel for multiple journeys as long as the total journey time is less than the duration of the planning period [13]. The Multi-Trip Vehicle Routing Problem (MTVRP) is an extension of the CVRP. Each vehicle can make multiple trips during its working period [14]. Fleischmann [15] is the first one to introduce MTVRP. He proposed the saving algorithm and used heuristics to determine a route. 
Taillard, et al. [16] proposed a three-phase algorithm to solve MTVRP. In the first phase, travel routes are designed to meet capacity constraints. In addition, these solutions are combined to achieve a particular CVRP. Finally, the route of each CVRP solution is assigned to the vehicle by solving the packing bin problem. Petch and Salhi [17] explained multi-phase heuristics that create several viable CVRP solutions and then solve them with bin packaging heuristics. Cattaruzza, et al. [18] presented a survey of MTVRP and routing problems related to multi-trip vehicles.

In many VRP studies, every customer is visited in a vehicle once a day. In fact, in practice, the customer takes more than one visit at a given time. Customers receive goods in several batches at other times due to limitations in the quantity of distribution. The routing problem associated with these conditions is a periodic vehicle routing problem (PVRP). A correct visit route is allocated to customers in PVRP [19]. Beltrami and Bodin [20] considered two heuristic algorithms to complete PVRP. The first algorithm used a saving algorithm to determine of daily route. The second idea was randomly to assign customers to delivery days and create ways for each day based on this assignment. Russell and Igo [21] proposed three heuristics for the problem. The first method was a clustering algorithm. The second algorithm took the routes developed by the first algorithm and improved them by a sequence of link exchanges. The third algorithm modified the savings algorithm by Clarke and Wright [22], where customers are allocated to days of the week by the delivery spacing constraints, and routes are formed using the savings algorithm. Christofides and Beasley [23] offered a heuristic that assigns customers to days and then solves the resulting daily VRPs.

Many PVRP variants have been developed, such as PVRP Time Windows by Cordeau, et al. [24]; Multi Depot PVRP [25]; Parthanadee and Logendran [26], PVRP Intermediate Facilities [27], and Site-dependent multi-trip PVRP [28]. To the best of our knowledge, only Alonso, et al. [28] have so far introduced Multi-Trip PVRP (MTPVRP). The objectives of this research are to propose procedures for solving problems Multi-Trip Periodic Vehicle Routing Problem. MTPVRP is a problem determining the route where the customers require a visit within one or more days of the planning period. The vehicle performs trips to the customers more than once per day. A hybrid approach was used to address these shortcomings by combining integer programming formulation and heuristics to achieve a better solution within an appropriate timeframe for computing. Because of the benefits of the cluster-first route-second (CFRS) heuristics approach to solve VRP, MTVRP, and PVRP explained above. The proposed CFRS is to solve the Multi-Trip Periodic Vehicle Routing Problem (MTPVRP). The drawbacks of this heuristic are that the solution is sub-optimal and that its efficiency depends heavily on the problem. The paper is organized as follows: section 1 is the introduction; section 2 describes methodology; section 3 explains the result and discussion, and section 4 is the conclusion.

\section{Methods}

\subsection{Problem description}

A depot makes deliveries to several customers using its vehicle. $I_{o}=\{0, \ldots, i, \ldots, n\}$ is the vertex set that consists of vertex subset $I=\{I, \ldots, i, \ldots, n\}$ which corresponds to the customers, and vertex 0, which corresponds to the depot. Each customer $i \in I$ corresponds known of non-negative delivery size $q_{i}$ whereas no delivery is required for 
the depot $\left(q_{0}=0\right)$. To serve these customers, a set of $v$ vehicles indexed by $K=$ $\{1, \ldots, k, \ldots v\}$ with identical capacity $C$, is available at the depot.

A trip is a sequence of customers starting from and finishing at the depot by a vehicle $k$ on the day $t$. A set of trips is denoted by $Z=\{1, \ldots, z, \ldots, n\}$, which means the number of trips that will be done by vehicle $k$ ranges from 1 to $n$, where $n$ is the number of customers. A set of days is denoted by $T=\{1, \ldots, t, \ldots, m\}$, where $m$ is the period length. A vehicle $k$ makes multiple trips when the total delivery quantity from all customers on day $t$ exceeds vehicle capacity. However, the total traveling time from those multiple trips has to be below the vehicle working hours. In this model, the number of trips performed by vehicle $k$ is a decision variable that is impacted by the fixed cost incurred.

During the period, each customer $i \in I$ is visited $1 \leq f_{i} \leq m$ times. Each customer $i \in I$ determines its visit frequency $f_{i}$. These visits must follow the set of allowable visitday combinations $S$. Table 1 shows the possible overall visit combination on each $f_{i}$ with period length $(\mathrm{m})$ equals to six. The six-digit sequence used in Table 1 represents the visit day from Monday to Saturday, where 1 shows there was a visit on that day and 0 vice versa.

Table 1. Visit frequency and visit-day combination on $m=6$ days

\begin{tabular}{ccccccc}
\hline $\mathrm{f}_{\mathrm{i}}$ & \multicolumn{6}{c}{$\mathrm{S}$} \\
\hline & 1 & 2 & 3 & 4 & 5 & 6 \\
\hline 1 & 100000 & 010000 & 001000 & 000100 & 000010 & 000001 \\
2 & 100100 & 010010 & 001001 & & & \\
3 & 101010 & 010101 & & & & \\
6 & 111111 & & & & & \\
\hline
\end{tabular}

\subsection{Proposed approach}

This section explains the cluster-first route-second (CFRS) heuristic approach used to solve the MTPVRP problem. The CFRS heuristics included in the two-phase algorithm category are as follows.

\section{Phase 1}

In phase 1 , customers were split into clusters. Customers within a cluster reached by vehicle- $k$ on day- $t$ with trip- $z$. The whole formulation and the branch-and-bound algorithm shall be used during the clustering stage to overcome the formulation. It is similar to research conducted by Fisher and Jaikumar [7] in which integer programming formulations are referred to as General Assignment Problems (GAP). GAP is used to determine which customers are included in each cluster.

The following is the notation that used an integer programming model:

$m$ period length

$c_{i k} \quad$ traveling cost from depot to customer $i$ with vehicle $k$ per unit of load

$f_{i} \quad$ visit frequency of customer $i$ during $m$-day period

$q_{i} \quad$ delivery size of customer- $i$ on any day

$C_{k} \quad$ maximum capacity of vehicle $k$

$f_{z} \quad$ fixed cost of trip $z$

$r_{z} \quad 1$ if trip $z$ is used 
$Y_{i t} \quad 1$ if customer $i$ was visited on the day $t, 0$ if otherwise

$X_{i t k z} \quad 1$ if customer $i$ was visited on the day $t$ by vehicle $k$ on trip $z, 0$ if otherwise

The model's objective is to minimize total cost, which is the sum of total traveling cost from depot to all customers and the total fixed cost of the vehicle. Total cost mathematically expressed in equation (1).

Minimize $\sum_{i \in I} \sum_{t \in T} \sum_{k \in K} \sum_{z \in Z} c_{i k} q_{i} X_{i t k z}+\sum_{z \in Z} f_{z} r_{z}$

Subject to

$\sum_{t \in T} Y_{i t}=f_{i} \quad i \in I$

$\sum_{r=t+1}^{r=\frac{m}{f_{i}}+t} Y_{i r}=1$

$i \in I ; t=0, \ldots, \ldots,\left(m-\frac{m}{f_{i}}\right)$

$\sum_{z \in Z} \sum_{k \in K} X_{i t k z}-Y_{i t}=0 \quad i \in I ; t \in T$

$\sum_{i \in I} q_{i} X_{i t k z} \leq C_{k}$

$t \in T ; k \in K ; z \in Z$

$\sum_{z \in Z} X_{i t k z} \leq n$

$i=0 ; t \in T ; k \in K$

$\sum_{z \in Z} X_{i t k z} \leq 1$

$i \in I ; t \in T ; k \in K$

$r_{z} \geq X_{i t k z}$

$i \in I ; t \in T ; k \in K ; z \in Z$

$y_{i t} \in\{0,1\}$

$i \in I ; t \in T$

$X_{i t k z} \in\{0,1\}$

$i \in I ; t \in T ; k \in K ; z \in Z$

Equation (6) guarantees that each customer has to visit $f$ times. Equation (7) ensures that each customer has to visit on the day that matches the visit frequency and visit-day combination, as explained in Table 1. Equation (8) guarantees that customer $i$ has to visited by vehicle $k$ with trip $z$ only if customer $i$ is to be visited on day $t$. Equation (9) assures that the total delivery size from all customers should not exceed the vehicle capacity. Equation (10) makes sure that each vehicle $k$ leaving the depot and perform multiple-trip in one day with a maximum of $n$ trips. On the contrary, Equation (11) makes sure that each vehicle $k$ can only leave customer $i$ once a day at maximum. Equation (12) guarantees that customer $i$ has to visit by vehicle $k$ with trip $z$ on day $t$ only if trip $z$ is used. Lastly, Equation (13) and (14) is set to make sure the decision variable is binary.

To determine $q_{i}$ in phase 1 , we refer to research conducted by Rusdiansyah \& Tsao, where they transformed an inventory routing problem inspired by the replenishment of vending machines into an instance of the PVRPTW [29]. Rusdiansyah \& Tsao computed a frequency for each customer based on a modified economic order quantity (EOQ) calculation and used these frequencies to define a PVRP. Given that each customer $i \in I$ has a demand rate $\lambda_{i}$ unit per day, during $m$-days period each customer have demands $D_{i}$, which is formulated in equation (11). 


$$
D_{i}=\lambda_{i} m \quad i \in I
$$

During the planning period, customer $i \in I$ requires replenishment of $f_{i}$ times with delivery quantity of $q_{1}, q_{2}, \ldots, q_{i r}, \ldots, q_{i f i}$. Let $u_{i r}$ be the interval between replenishment $r$ and replenishment $r+1$ for customer $i \in I$, the delivery size $q_{i r}$ can be formulated in equation (12).

$$
q_{\text {ir }}=\lambda_{i} u_{i r} \quad i \in I ; r=1, \ldots, f_{i}
$$

Since some improvements in algorithms adjust the visit-day combination and the frequency of visits, it is crucial to ensure that these changes do not impact the optimal cost of inventory holdings. A stationary interval property was used to obtain an equation (13) and (14).

$$
\begin{array}{ll}
u_{i 1}=u_{i 2}=\cdots=u_{i f_{i}}=\frac{m}{f_{i}} & i \in I \\
q_{i}=q_{i r}=\lambda \frac{m}{f_{i}}=\frac{D_{i}}{f_{i}} & i \in I ; r=1, \ldots, f_{i}
\end{array}
$$

\section{Phase 2}

Phase 2 sets out the cluster route and verifies that the total travel time for all trips does not exceed the working hours of the vehicle. Since the day of the visit and the vehicle for each cluster has been established. Therefore the route determination belongs to Traveling Salesman Problem (TSP). The researchers propose the tabu search algorithm as it is one of the metaheuristics commonly used to resolve TSP. The steps performed in phase 2 are as follows:

1. Based on the 1st phase solution, plan the visit route on each cluster route using Tabu Search Algorithm. First of all, determine the initial route randomly. Identify the neighborhood collection by swapping two customers, sliding the next customer, and flipping the customer series to obtain new paths. Next, pick the best neighborhood movement from the shortest route distance and by updating the Tabu list. If the solution is not tabu, then the move is performed, but if it is tabu, check if aspiration criteria are met. The neighborhood set search is repeated and stopped after the maximum iteration is fulfilled.

2. Calculate the total distance traveled and the total time traveled on each cluster path. Instead, count the cumulative length of each cluster by summing up the total journey time and the time taken during the service to each customer.

3. Calculate the total duration of each vehicle per day by summing up the total duration of each cluster on that day and the average service time of the dealer.

4. Compare the total time of the vehicle per day with the working hours of the vehicle. Suppose the cumulative length of the journey is less than the working hours. In that case, the researchers infer that the cluster route is feasible. Otherwise, the route created will not be feasible and will not be included in the next phase.

5. Based on feasible cluster routes, compare the total travel distance or total transport cost for each planning period and define $\mathrm{f}$ (visit frequency number), which gives the shortest total travel distance or minimum transport cost. 


\subsection{Data collecting}

The application of the CFRS method was carried out in actual cases by the LPG manufacturer. Therefore, the data was gathered by interviews and findings with the distributor. Distributor (D) delivered LPG to 29 retailers (T1, T2,.., T29) using two vehicles, each with a capacity of 150 cans. Observation results showed that the manufacturer splits retailers into two distribution areas by the number of vehicles. The dealer usually delivers two days a week to vehicle one distributor three times a week to vehicle 2 . Vehicles also took more than one trip a day due to capacity limitations. As a result, this distributor's route determination problem fits into the Multi-Trip Periodic Vehicle Routing Problem (MTPVRP). The total distance traveled and the time traveled per cluster were measured using Google Maps. Retailers are relatively close, with travel distances of between $0.058-16.19 \mathrm{~km}$ and $4.37 \mathrm{~km}$ on average. From field observation, the loading and unloading time will take 30 seconds. The loading and unloading time for each trip was obtained by assuming that the vehicle was fully loaded with 150 cans. Thus the average service time for 150 LPG cans was 75 minutes or 1.25 hours.

\subsection{Experimental procedure}

In phase 1 , three visit frequency $\left(f_{i}=2,3\right.$, and 6$)$ were used to generate several solution scenarios. In each scenario, the solutions were formed using the mathematical model explained in phase 1 and setting the right-hand side value of equation (6) to the frequency value of the scenario. Based on the value of the decision, variable $X_{i t k z}$, the cluster members are obtained by grouping customers with the same $t, k$, and $z$ index combination. Therefore, the solution for each scenario was the number of clusters and the customers in each cluster. The solution scenarios from phase 1 are input to phase 2 by ignoring the total cost obtained from the mathematical model solution. It was done because traveling time and traveling distance between customers are disregarded. Thus the total cost only showed traveling costs from the distributor. In phase 2 , the solution chosen was the scenario with $f_{i}$ that generated the lowest total traveling distance.

\section{Result and Discussion}

Depending on the path of the current dealer, the number of LPG cans to be delivered is 3,580 cans per week for vehicles 1 and 2 . The number of route clusters by both vehicles is 24 . The total distance traveled and the total time per day of both vehicles for one week is $377.44 \mathrm{~km}$ and 72.41 hours. By using the proposed CFRS approach, three solution scenarios are generated. The solution at $f_{i}=2$ is shown in Table 2 . It can be seen that there are 29 clusters to be taken by two vehicles for one week. The members of cluster 1 include consumer T2,T10, and T12. They are visited on trip 1 of Monday using vehicle 1.

Furthermore, T16 and T26 are in cluster 2 and are visited on trip 1 of Monday using vehicle one. Compared to the distributor's route, the number of clusters was five times more than the number of clusters for the distributor's method. It shows an increase in total loading and unloading time at the distributor compared to before. The total load of both vehicles is 3,580 cans, equal to the total number of cans of the current distributor's route. 
Table 2. Traveling distance, traveling time, and total duration time calculation for solution scenario in $f_{i}=2$

\begin{tabular}{|c|c|c|c|c|c|c|c|}
\hline & & & hicle 1 & & & & \\
\hline Day and Trip & $\begin{array}{l}\text { Number and } \\
\text { Route of each } \\
\text { cluster }\end{array}$ & Load & $\begin{array}{c}\text { Total } \\
\text { traveling } \\
\text { distance } \\
(\mathrm{km})\end{array}$ & $\begin{array}{l}\text { Total } \\
\text { traveling } \\
\text { time } \\
\text { (hour) }\end{array}$ & $\begin{array}{c}\text { Total } \\
\text { service } \\
\text { time } \\
\text { (hour) }\end{array}$ & $\begin{array}{c}\text { Total } \\
\text { duration } \\
\text { time per } \\
\text { cluster } \\
\text { (hour) }\end{array}$ & $\begin{array}{c}\text { Total } \\
\text { duration } \\
\text { time per } \\
\text { day } \\
\text { (hour) }\end{array}$ \\
\hline Monday trip1 & (1) D-T2-T12-T10-D & 150 & 12.03 & 0.40 & 1.25 & 1.65 & \\
\hline Monday trip 2 & (2) D-T26-T16-D & 130 & 13.69 & 0.46 & 1.08 & 1.54 & 8.37 \\
\hline Monday trip 3 & (3) D-T25-T19-D & 135 & 9.23 & 0.31 & 1.13 & 1.43 & \\
\hline Tuesday trip 1 & (4) D-T11-T9-D & 100 & 11.93 & 0.40 & 0.83 & 1.23 & \\
\hline Tuesday trip 2 & (5) D-T6-T5-T4-D & 150 & 7.31 & 0.24 & 1.25 & 1.49 & 7.62 \\
\hline Tuesday trip 3 & (6) D-T18-D & 105 & 8.22 & 0.27 & 0.88 & 1.15 & \\
\hline Wednesday trip 1 & (7) D-T13-T8-D & 95 & 9.24 & 0.31 & 0.79 & 1.10 & \\
\hline Wednesday trip 2 & (8) D-T3-T7-D & 100 & 11.20 & 0.37 & 0.83 & 1.21 & 7.55 \\
\hline Wednesday trip 3 & (9) D-T28-T21-D & 135 & 11.14 & 0.37 & 1.13 & 1.50 & \\
\hline Thursday trip1 & (10) D-T2-T16-D & 105 & 10.09 & 0.34 & 0.88 & 1.21 & \\
\hline Thursday trip 2 & (11) D-T26-T19-D & 135 & 12.69 & 0.4 & & 1.55 & 7.70 \\
\hline Thursday trip 3 & (12) D-T12-T10-D & 100 & 10.66 & 0.36 & 0.83 & 1.19 & \\
\hline Friday trip 1 & (13) D-T11-T9-T5-D & 150 & 16.73 & 0.56 & 1.25 & 1.81 & \\
\hline Friday trip 2 & (14) D-T27-T4-D & 125 & 9.01 & 0.30 & 1.04 & 1.34 & 8.39 \\
\hline Friday trip 3 & (15) D-T20-T24-D & 135 & 10.93 & 0.36 & 1.13 & 1.49 & \\
\hline Saturday trip 1 & (16) D-T1-T8-D & 100 & 6.68 & 0.22 & 0.83 & 1.06 & \\
\hline Saturday trip 2 & (17) D-T3-T28-D & 110 & 6.69 & 0.22 & 0.92 & 1.14 & 4.70 \\
\hline Total per n & reek vehicle 1 & 2060 & 177.47 & 5.92 & 17.17 & 23.08 & 44.33 \\
\hline & & & ehicle 2 & & & & \\
\hline Day and Trip & $\begin{array}{l}\text { Number and route } \\
\text { of each cluster }\end{array}$ & Load & $\begin{array}{c}\text { Total } \\
\text { traveling } \\
\text { distance } \\
(\mathrm{km})\end{array}$ & $\begin{array}{l}\text { Total } \\
\text { traveling } \\
\text { time } \\
\text { (hour) }\end{array}$ & $\begin{array}{c}\text { Total } \\
\text { service } \\
\text { time } \\
\text { (hour) }\end{array}$ & $\begin{array}{c}\text { Total } \\
\text { duration } \\
\text { time per } \\
\text { cluster } \\
\text { (hour) }\end{array}$ & $\begin{array}{c}\text { Total } \\
\text { duration } \\
\text { time per } \\
\text { day } \\
\text { (hour) }\end{array}$ \\
\hline Monday trip1 & (18) D-T17-T23-D & 140 & 5.68 & 0.19 & 1.17 & 1.36 & 2.61 \\
\hline Tuesday trip 1 & (19) D-T20-T29-D & 150 & 8.46 & 0.28 & 1.2 & 1.53 & \\
\hline Tuesday trip 2 & (20) D-T22-DD & 90 & 10.94 & 0.36 & 0.75 & 1.11 & 7.95 \\
\hline Tuesday trip 3 & (21) D-T27-24-D & 150 & 9.01 & 0.30 & 1.25 & 1.55 & \\
\hline Wednesday trip 1 & (22) D-T1-T15-D & 100 & 3.14 & 0.10 & 0.83 & 0.94 & 2.19 \\
\hline Thursday trip1 & (23) D-T14-T17-D & 140 & 8.82 & 0.29 & 1.17 & 1.46 & \\
\hline Thursday trip 2 & (24) D-T25-T23-D & 135 & 10.52 & 0.35 & 1.13 & 1.48 & 5.44 \\
\hline Friday trip 1 & (25) D-T22-D & 90 & 10.94 & 0.36 & 0.75 & 1.11 & \\
\hline Friday trip 2 & (26) D-T18-D & 105 & 8.22 & 0.27 & 0.88 & 1.15 & 7.50 \\
\hline Friday trip 3 & (27) D-T6-T29-D & 140 & 9.51 & 0.32 & 1.17 & 1.48 & \\
\hline Saturday trip 1 & (28) D-T14-T21-D & 135 & 10.47 & 0.35 & 1.13 & 1.47 & \\
\hline Saturday trip 2 & (29) D-T7-T13-T15-D & 145 & 10.27 & 0.34 & 1.21 & 1.55 & \\
\hline Total per $\mathrm{w}$ & eek vehicle 2 & 1520 & 105.98 & 3.53 & 12.67 & 16.20 & 31.20 \\
\hline Total per weel & vehicle 1 and 2 & 3580 & 283.45 & 9.45 & 29.83 & 39.28 & 75.53 \\
\hline
\end{tabular}

The cluster route and the cumulative length of the day are obtained by following steps 1-4 in phase 2. The cluster route formed by the tabu search algorithm can be seen in Table 2. From the proposed route, the total traveling distance of both vehicles is $283.45 \mathrm{~km} /$ week, which is a reduction of $93.99 \mathrm{~km} /$ week compared to the distributor's route. Since the average distance between retailers is only $4.37 \mathrm{~km}$, this reduction is significant enough. It shows that step 2 generates a more efficient route, even though the number of clusters is higher than the initial route. The shorter total travel distance also 
results in a shorter total duration per cluster. Concerning the cumulative length of each node, the suggested path is 3.13 hours shorter than the route of the distributor (from 42.41 to 39.28). By comparison, the average length of the day decreases by 3.12 hours according to the dealer's path. It is because the estimated number of clusters is higher, so the loading and unloading time is longer. However, this additional time is still feasible as none of the working hours per day is greater than 9 hours.

Table 3 shows a comparison of the total travel distance, the total travel time, the total duration of each day, and the number of clusters in each scenario. Total travel distance and total travel time are the highest at $\mathrm{fi}=6$ because every customer is visited up to 6 times a week. On the opposite, at $\mathrm{fi}=2$, the total distance traveled and the total time traveled is the lowest of the other frequencies. As explained above, the number of clusters tends to impact the total duration of the service at the distributor per day. However, the cumulative cycle time at $\mathrm{fi}=6$ is longer than $\mathrm{fi}=2$ even though the number of clusters is smaller. It is because the travel time at $\mathrm{fi}=6$ is longer, although the service time at the dealer is shorter. Based on a calculation of the total distance traveled and the total length per day, the solution scenario with $\mathrm{fi}=2$ is chosen. The proposed route for distributors is set out in Table 2.

Table 3. Comparison of total traveling distance, total traveling time, and total duration time per day for each visit frequency scenario

\begin{tabular}{ccccc}
$\begin{array}{c}\text { Visit } \\
\text { frequency } \\
\text { scenario }\end{array}$ & $\begin{array}{c}\text { Number of } \\
\text { clusters }\end{array}$ & $\begin{array}{c}\text { Total traveling } \\
\text { distance }(\mathrm{km} / \text { week) }\end{array}$ & $\begin{array}{c}\text { Total traveling } \\
\text { time (hour/week) }\end{array}$ & $\begin{array}{c}\text { Total duration } \\
\text { time per day } \\
\text { (hour/week) }\end{array}$ \\
\hline 2 & 29 & 283.45 & 9.45 & 75.53 \\
\hline 3 & 36 & 441.03 & 14.70 & 89.53 \\
\hline 6 & 24 & 483.9 & 16.16 & 75.98 \\
\hline
\end{tabular}

The application of the CFRS heuristics approach to the MTPVRP solution has shown that the procedures have generated a better route solution. There was a decrease in travel distance of $93.99 \mathrm{~km} /$ week, equivalent to a saving of 24.9 percent. First, by comparing the total distance travel reduction to the cost of fuel, by assuming that 1 liter of Petalite can be used to travel $6 \mathrm{~km}$ and that the price of Pertalite is IDR 7,600/liter, savings of IDR 119,053 per week or IDR 6,190,740 per year have been made. These results support the research conducted by Saputro and Prihatina [30], which demonstrates CFRS to reduce the total traveling distance and fuel cost.

\section{Conclusion}

The objectives of this research are to propose procedures for solving problems Multi-Trip Periodic Vehicle Routing Problem. A hybrid approach was used to address these shortcomings by combining integer programming formulation and heuristics. Applying the proposed solution on a case-by-case basis concluded that the cluster-first route-second (CFRS) heuristic was able to generate a better route based on the total distance and fuel costs. Points to enhance further work are: 1) Create a mathematical model in phase 1 so that the frequency is part of the decision variable instead of the trial-error; 2) Model a tab search program using VBA Macro Excel in phase 2 because the lingo performance of the phase 1 result can be stored in excel. 


\section{References}

[1] G. B. Dantzig and J. H. Ramser, "The truck dispatching problem," Management science, vol. 6, pp. 80-91, 1959. https://doi.org/10.1287/mnsc.6.1.80.

[2] B. Eksioglu, A. V. Vural, and A. Reisman, "The vehicle routing problem: A taxonomic review," Computers \& Industrial Engineering, vol. 57, pp. 1472-1483, 2009. https://doi.org/10.1016/j.cie.2009.05.009.

[3] C. Lin, K. L. Choy, G. T. Ho, S. H. Chung, and H. Lam, "Survey of green vehicle routing problem: past and future trends," Expert systems with applications, vol. 41, pp. 1118-1138, 2014. https://doi.org/10.1016/j.eswa.2013.07.107.

[4] S. Srivatsa Srinivas and M. Gajanand, "Vehicle routing problem and driver behaviour: a review and framework for analysis," Transport reviews, vol. 37, pp. 590-611, 2017. https://doi.org/10.1080/01441647.2016.1273276.

[5] A. Imran and L. Okdinawati, "Adaptation Of The Variable Neighborhood Search Heuristic To Solve The Vehicle Routing Problem," Jurnal Teknik Industri, vol. 12, pp. 10-15, 2012. https://doi.org/10.22219/JTIUMM.Vol12.No1.10-15.

[6] B. E. Gillett and L. R. Miller, "A heuristic algorithm for the vehicle-dispatch problem," Operations research, vol. 22, pp. 340-349, 1974. https://doi.org/10.1287/opre.22.2.340.

[7] M. L. Fisher and R. Jaikumar, "A generalized assignment heuristic for vehicle $\begin{array}{llllll}\text { routing," Networks, } & \text { vol. } 11, \quad \text { pp. } & 109-124, & 1981 .\end{array}$ https://doi.org/abs/10.1002/net.3230110205.

[8] R. Dondo and J. Cerdá, "A cluster-based optimization approach for the multidepot heterogeneous fleet vehicle routing problem with time windows," European Journal of Operational Research, vol. 176, pp. 1478-1507, 2007. https://doi.org/10.1016/j.ejor.2004.07.077.

[9] K. Shin and S. Han, "A centroid-based heuristic algorithm for the capacitated vehicle routing problem," Computing and Informatics, vol. 30, pp. 721-732, 2012. http://www.cai.sk/ojs/index.php/cai/article/viewArticle/192.

[10] F. Cakir, W. N. Street, and B. W. Thomas, "Revisiting Cluster First, Route Second for the Vehicle Routing Problem," no. August, 2015.

[11] S. E. CÖMERT, H. R. YAZGAN, I. Sertvuran, and H. ŞENGÜL, "A new approach for solution of vehicle routing problem with hard time window: an application in a supermarket chain," Sādhanā, vol. 42, pp. 2067-2080, 2017. https://doi.org/10.1007/s12046-017-0754-1.

[12] D. Hiquebran, A. Alfa, J. Shapiro, and D. Gittoes, "A revised simulated annealing and cluster-first route-second algorithm applied to the vehicle routing problem," Engineering Optimization, vol. 22, pp. 77-107, 1993. https://doi.org/10.1080/03052159308941327.

[13] D. A. P. Putri, "Vehicle Routing Problem Dengan Time Window Untuk Multiple Product Dan Multiple Route Menggunakan Algoritma Sequential Insertion," Jurnal Teknik Industri, vol. 17, pp. 22-30, 2016. https://doi.org/10.22219/JTIUMM.Vol17.No1.22-30.

[14] A. Mingozzi, R. Roberti, and P. Toth, "An exact algorithm for the multitrip vehicle routing problem," INFORMS Journal on Computing, vol. 25, pp. 193-207, 2013. https://doi.org/10.1287/ijoc.1110.0495.

[15] B. Fleischmann, "The vehicle routing problem with multiple use of vehicles," Fachbereich Wirtschaftswissenschaften, Universität Hamburg, 1990. 
[16] É. D. Taillard, G. Laporte, and M. Gendreau, "Vehicle routeing with multiple use of vehicles," Journal of the Operational research society, vol. 47, pp. 1065-1070, 1996. https://doi.org/10.1057/jors.1996.133.

[17] R. J. Petch and S. Salhi, "A multi-phase constructive heuristic for the vehicle routing problem with multiple trips," Discrete Applied Mathematics, vol. 133, pp. 69-92, 2003. https://doi.org/10.1016/S0166-218X(03)00434-7.

[18] D. Cattaruzza, N. Absi, and D. Feillet, "Vehicle routing problems with multiple trips," 4OR, vol. 14, pp. 223-259, 2016. https://doi.org/10.1007/s10288-016-0306-2.

[19] A. M. Campbell and J. H. Wilson, "Forty years of periodic vehicle routing," Networks, vol. 63, pp. 2-15, 2014. https://doi.org/10.1002/net.21527.

[20] E. J. Beltrami and L. D. Bodin, "Networks and vehicle routing for municipal waste collection," Networks, vol. 4, pp. 65-94, 1974. https://doi.org/10.1002/net.3230040106.

[21] R. Russell and W. Igo, "An assignment routing problem," Networks, vol. 9, pp. 117, 1979. https://doi.org/abs/10.1002/net.3230090102.

[22] G. Clarke and J. W. Wright, "Scheduling of vehicles from a central depot to a number of delivery points," Operations research, vol. 12, pp. 568-581, 1964. https://doi.org/10.1287/opre.12.4.568.

[23] N. Christofides and J. E. Beasley, "The period routing problem," Networks, vol. 14, pp. 237-256, 1984. https://doi.org/10.1002/net.3230140205.

[24] J.-F. Cordeau, G. Laporte, and A. Mercier, "A unified tabu search heuristic for vehicle routing problems with time windows," Journal of the Operational research society, vol. 52, pp. 928-936, 2001. https://doi.org/10.1057/palgrave.jors.2601163.

[25] J. F. Cordeau, M. Gendreau, and G. Laporte, "A tabu search heuristic for periodic and multi-depot vehicle routing problems," Networks: An International Journal, vol. 30, pp. 105-119, $1997 . \quad$ https://doi.org/10.1002/(SICI)10970037(199709)30:2<105::AID-NET5>3.0.CO;2-G.

[26] P. Parthanadee and R. Logendran, "Periodic product distribution from multidepots under limited supplies," Iie Transactions, vol. 38, pp. 1009-1026, 2006. https://doi.org/10.1080/07408170600575454.

[27] E. Angelelli and M. G. Speranza, "The periodic vehicle routing problem with intermediate facilities," European journal of Operational research, vol. 137, pp. 233-247, 2002. https://doi.org/10.1016/S0377-2217(01)00206-5.

[28] F. Alonso, M. J. Alvarez, and J. E. Beasley, "A tabu search algorithm for the periodic vehicle routing problem with multiple vehicle trips and accessibility restrictions," Journal of the Operational Research Society, vol. 59, pp. 963-976, 2008. https://doi.org/10.1057/palgrave.jors.2602405.

[29] A. Rusdiansyah and D.-b. Tsao, "An integrated model of the periodic delivery problems for vending-machine supply chains," Journal of Food Engineering, vol. 70, pp. 421-434, 2005. https://doi.org/10.1016/j.jfoodeng.2004.05.073.

[30] T. E. Saputro and A. Prihatina, "Perencanaan Jadwal dan Rute Distribusi Rokok untuk Menekan Total Biaya Transportasi," Jurnal Teknik Industri, vol. 13, pp. 151-157, 2012. https://doi.org/10.22219/JTIUMM.Vol13.No2.151-157. 